\title{
Evaluación de la extracción de ácidos grasos a partir de cabezas de sardina (Opisthonema libertate) subproducto de la industria pesquera
}

\author{
(Evaluation of extraction of fatty acids from sardine's head \\ (Opisthonema libertate) by-product of fishing industry)
}

\author{
Belén Carrillo ${ }^{1}$, Mauricio Mosquera ${ }^{1}$
}

\begin{abstract}
Resumen:
Los ácidos grasos poliinsaturados son compuestos bioactivos utilizados como suplementos nutricionales en la industria alimentaria y farmacéutica, presentes en peces azules como la sardina (Opisthonema libertate). El objetivo de la investigación fue la recuperación de ácidos grasos a partir de las cabezas de sardina. Se realizó una hidrólisis básica con la finalidad de extraer el aceite presente. Como agente hidrolizante se utilizó $\mathrm{NaOH}$ en cuatro concentraciones, el mayor porcentaje de recuperación de aceite saponificado correspondió a un 3,64 \%, con una concentración $1 \mathrm{M}$ de $\mathrm{NaOH}$. La utilización de esta base en la extracción, permitió simultáneamente saponificar el aceite recuperado. Se analizó la influencia de factores como la temperatura y la concentración de $\mathrm{H}_{2} \mathrm{SO}_{4}$ en el aislamiento de los ácidos grasos mediante el proceso de acidulación. La mejor condición experimental del proceso se dio a $20 \stackrel{\circ}{\circ}$ y $75 \%$ de concentración de $\mathrm{H}_{2} \mathrm{SO}_{4}$. El análisis de ácidos grasos se realizó mediante cromatografía de gases, donde se determinó una concentración de $2233,70 \mu \mathrm{g} / \mathrm{m}$, de los cuales el $82,55 \%$ correspondió a ácidos grasos saturados, el 13,72 \% a ácidos monoinsaturados y el 3,73\% para ácidos poliinsaturados. Dentro de los ácidos grasos poliinsaturados se destacó la presencia del ácido eicosapentaenoico EPA (omega-3) con una concentración de 73,12 $\mu \mathrm{g} / \mathrm{mL}$.
\end{abstract}

Palabras clave: ácidos grasos; hidrólisis; omega-3; saponificación; sardina.

\begin{abstract}
:
Polyunsaturated fatty acids are bioactive compounds employed as nutritional supplements in the food and pharmaceutical industry, those are present in blue fish like sardine (Opisthonema libertate). The purpose of this investigation was the recovery of these acids from sardine heads. A basic hydrolysis was carried out in order to extract the oil. $\mathrm{NaOH}$ was the hydrolyzing agent, tested in four concentrations. As a result, $3.64 \%$ was the highest percentage of saponified oil recovery, with a concentration of $1 \mathrm{M} \mathrm{NaOH}$. This base allowed simultaneously saponify the recovered oil in the extraction. The influence of temperature and the concentration of $\mathrm{H} 2 \mathrm{SO} 4$ in the isolation of fatty acids by the acidulation process was analyzed. The best experimental condition of the process was given at $20^{\circ} \mathrm{C}$ and $75 \%$ of $\mathrm{H} 2 \mathrm{SO} 4$ concentration in the extracted oil, it was determined $2233.70 \mu \mathrm{g} / \mathrm{mL}$ of fatty acids, where $82.55 \%$ corresponded to saturated fatty acids, $13.72 \%$ to monounsaturated acids and $3.73 \%$ to polyunsaturated acids. Among the polyunsaturated fatty acids, was emphasized the presence of eicosapentaenoic acid EPA (omega-3) with a concentration of $73.12 \mu \mathrm{g} / \mathrm{mL}$.
\end{abstract}

Keywords: fatty acids; hydrolysis; omega-3; saponification; sardine

\footnotetext{
${ }^{1}$ Escuela Politécnica Nacional, Quito, Ecuador ( \{maria.carrillo01, mauricio.mosquera\} @epn.edu.ec )
} 


\section{Introducción}

En Ecuador la capacidad de producción de sardinas en conserva es de aproximadamente 40 mil toneladas anuales, de las cuales solo el $86 \%$ es destinado al consumo humano; mientras que el $14 \%$ restante corresponde a desperdicios como cabezas, colas y vísceras (FAO, 2014; ProEcuador, 2015). Estos desperdicios son comúnmente desechados al océano, práctica que genera un impacto ambiental (MAE, 2013). En el mejor de los casos, los desperdicios de la industria sardinera, son utilizados para la elaboración de productos de bajo valor agregado como harina de pescado, de la cual se obtiene aceite, que suele ser utilizado para la elaboración de alimento de animales de criadero (IFFO, 2008). Sin embargo, en la actualidad se conoce que dicho aceite es la principal fuente de ácidos grasos esenciales de origen animal, los cuales son utilizados para la elaboración de suplementos nutricionales, en la dieta de seres humanos (Sanz, 2009; Valenzuela et al., 2012).

Los ácidos grasos son ácidos carboxílicos cuya cadena puede ser saturada o insaturada. En la cadena saturada, todos sus enlaces son simples; mientras que, la insaturada se caracteriza por la presencia de enlaces dobles (Gargaud, 2011). Los ácidos grasos que no pueden ser sintetizados en el cuerpo humano, reciben el nombre de ácidos grasos esenciales y también son conocidos como ácidos grasos poliinsaturados (Gellman y Turner, 2013; Mora, 2002). En los procesos metabólicos de los seres humanos, estos compuestos son los responsables de otorgar soporte estructural a los fosfolípidos de las membranas celulares y debido a la ausencia en el organismo de los ácidos grasos cuyo doble enlace se encuentre en la posición omega-3 y omega-6, estos deben ser ingeridos en los alimentos (Gatica, 2011; Valenzuela et al., 2011). El consumo de estos ácidos produce además beneficios a la salud, entre los que se destaca la prevención de enfermedades cardiovasculares, artritis reumatoide, deterioro cognitivo y diversos tipos de cáncer (Larsen et al., 2011). Este tipo de beneficios han sido comprobados en diversos estudios realizados en países como Japón, donde el consumo de pescados ricos en omega-3 y omega- 6 es elevado y como consecuencia se evidencia una disminución significativa de enfermedades cardiovasculares, en comparación con otras dietas que poseen una baja ingesta de estos ácidos grasos esenciales (Iso et al., 2006).

Implementar métodos, que permitan la extracción y purificación de estos ácidos, representa una alternativa para el desarrollo de nuevos productos a nivel nacional, con los cuales además sea posible incursionar en mercados emergentes. Existen varios estudios enfocados en el desarrollo de técnicas para la extracción de ácidos grasos, a partir de subproductos de la industria pesquera. Algunos métodos enfocan su estudio en el uso de lipasas, enzimas que debido a su selectividad, permiten extraer los ácidos grasos presentes en el aceite, por la ruptura de enlaces específicos (Valverde et al., 2013). Existen otros métodos de purificación mediante solventes orgánicos, donde la diferencia de polaridad y configuración espacial son la base de la extracción (Caprioli et al., 2016). 
Otros métodos como la extracción con fluidos supercríticos, separan los ácidos grasos debido a la polaridad de los mismos en el medio (Rubio et al., 2012; Sahena et al., 2009).

En este estudio se plantea la extracción del aceite por medio de una hidrólisis básica con hidróxido de sodio, en la cual la temperatura, fuerza iónica, $\mathrm{pH}$ y polaridad del disolvente actúan como agentes desnaturalizantes que desestabilizan la proteína transformándola en aminoácidos, los mismos que al ser solubles en agua se separan del aceite debido a la inmisibilidad entre dichas soluciones, de esta manera se liberan los lípidos de la proteína (Giraldo et al., 2010; Macarulla y Goñi, 2002; Velásquez, 2006).

De manera simultánea al proceso previamente descrito, el $\mathrm{NaOH}$ al ser un álcali altamente reactivo, cuando se encuentra en solución, entra en contacto con los lípidos aislados, reacciona con estos y los saponifica, como se observa en la Figura 1. Como producto de este proceso se obtiene una sal asociada al ácido graso y glicerina como subproducto (Mercantili et al., 2012; Nolsøe y Undeland, 2009; Wijayarathne y Wasalathilake, 2014).

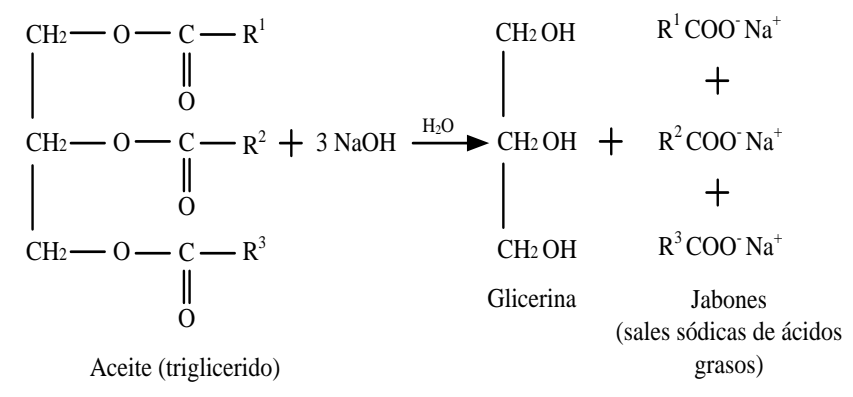

Figura 1. Hidrólisis básica de un triglicérido

(McMurry, 2012)

Las sales de ácidos grasos contienen los ácidos grasos que serán aislados por medio de un proceso de acidulación, el cual utiliza ácido sulfúrico como reactivo disociante. La acción catalítica del ácido permite la ruptura de los enlaces en las sales de manera que los ácidos grasos son liberados de la molécula. En este proceso, el sodio asociado al ácido graso es remplazado por un ion $\mathrm{H}^{+}$. $\mathrm{La}$ sustitución de minerales como el sodio en triglicéridos saponificados se da gracias a la donación de un protón por parte del ácido utilizado, con la consecuente formación de sulfato de sodio como subproducto tal como se observa en la Figura 2 (Gargaud, 2011; Nielsen, 2003; Yúfera, 2007).

Para poder analizar los ácidos grasos existentes en el aceite, estos deben ser esterificados ya que los ésteres de los ácidos grasos poseen en su estructura un grupo metilo que le otorga menor polaridad y como consecuencia, los vuelve más volátiles, lo que permite la identificación y cuantificación de los mismos mediante cromatografía de gases (Gracia, 2010). La separación dentro del equipo se da por la afinidad que presenta la muestra entre la fase móvil que representa el gas portador y la estacionaria que consiste en la columna de la cromatografía (Karasek y Clement, 2012). 


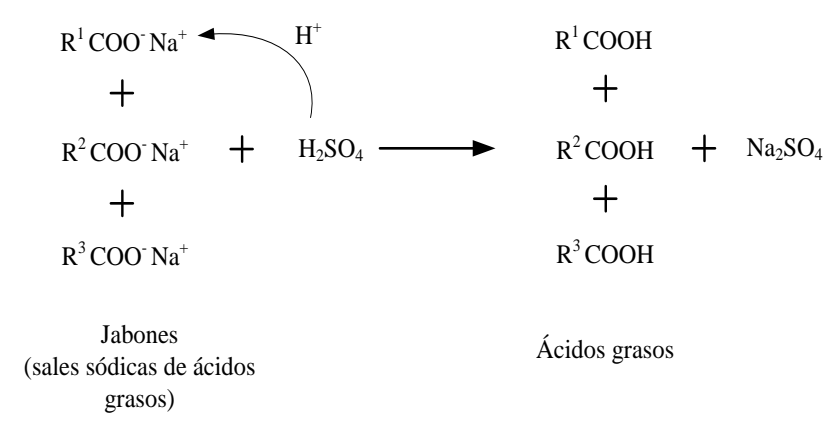

Figura 2. Acidulación del aceite saponificado

(Cermak et al., 2012; Scrimgeour, 2005)

En síntesis, la presente investigación tiene como objetivo obtener ácidos grasos a partir de cabezas de sardina (Opisthonema libertate), mediante hidrólisis básica y posterior acidulación de las sales sódicas de los ácidos grasos. Este estudio se presenta como una alternativa para la revalorización de residuos de la industria sardinera.

\section{Metodología}

\subsection{Materia prima}

La materia prima que se utilizó para el desarrollo del presente estudio provino de los residuos de la actividad pesquera de la localidad Crucita $\left(0^{\circ} 52^{\prime} \mathrm{S} ; 80^{\circ} 32^{\prime} \mathrm{O}\right)$ ubicada a 0 m.s.n.m. en la costa ecuatoriana de la provincia de Manabí. Se emplearon $10 \mathrm{~kg}$ de cabezas de sardina, especie de pelágicos conocidos coloquialmente con el nombre de pinchagua (Opisthonema libertate). La materia prima fue congelada hasta su uso.

\subsection{Caracterización de la materia prima}

El contenido de humedad se determinó mediante el método 950.46 de la AOAC (2007), para lo cual se utilizó una estufa marca MEMMERT con control de temperatura y tiempo. Se determinó el contenido de cenizas mediante el método 920.153 de la AOAC (2007), para lo cual se empleó una mufla marca LINDBERG, modelo BF51728C-1. Para la determinación del contenido de proteína se empleó el método 981.10 de la AOAC (2007), con la ayuda de un destilador marca GERHARDT, modelo Vapodest 45s, en conjunto con un digestor. Finalmente, el porcentaje de extracto etéreo se determinó de acuerdo con lo descrito en el método 960.39 de la AOAC (2007), mediante un extractor de grasas con solventes marca VELP, modelo SER 148.

\subsection{Hidrólisis básica para la recuperación de aceite saponificado}

El proceso de hidrólisis básica se realizó de acuerdo con el método descrito por Hordur y Necla (2003), con una modificación en la variación de la concentración de $\mathrm{NaOH}$, la cual se llevó a cabo de acuerdo con un diseño experimental al azar con tres repeticiones en el que se utilizaron valores de 0,$05 ; 0,10 ; 0,50$ y $1,00 \mathrm{M}$. Con base en experimentaciones previas, se pesaron aproximadamente 
$500 \mathrm{~g}$ de materia prima en una balanza marca OHAUS con capacidad de $6 \mathrm{~kg} \mathrm{y}$ 0,001 $\mathrm{kg}$ de sensibilidad. Estas cabezas fueron colocadas en un vaso de precipitación de $1 \mathrm{~L}$ de capacidad, al cual se agregaron lentamente $300 \mathrm{~mL}$ de cada una de las soluciones de $\mathrm{NaOH}$, de manera que las cabezas quedaron totalmente cubiertas. A continuación, se calentaron hasta una temperatura de $70^{\circ} \mathrm{C}$, con agitación constante de 70 oscilaciones por minuto, durante $2 \mathrm{~h}$, en un baño térmico marca Selecta modelo Unitronic Or (Harting et al., 2010).

Como resultado de la hidrólisis básica se obtuvo aceite saponificado, hidrolizado de proteína y residuos sólidos. Posteriormente, los residuos sólidos que incluyeron espinas, se separaron de la solución mediante la retención de las mismas en un cedazo convencional, con una abertura máxima de $1 \mathrm{~mm}$. Luego, la solución obtenida se colocó en un embudo de separación y se dejó en reposo, de modo que por decantación, se formaron dos fases, de las cuales, la superior conocida como aceite saponificado compuesto por las sales sódicas de ácidos grasos, fue separada, mientras que en la parte inferior se retuvieron residuos sólidos no deseados como arena y una fase acuosa constituida principalmente por hidrolizado de proteína.

El aceite saponificado se procesó en una centrífuga marca Thermo modelo HN SII a 2500 rpm, durante $10 \mathrm{~min}$, para separar cualquier residuo sólido remanente (Jayasinghe et al., 2013). Finalmente, el aceite saponificado separado y centrifugado se pesó en una balanza marca Boeco con capacidad de $200 \mathrm{~g}$ y sensibilidad de $0,1 \mathrm{mg}$. Posteriormente, se calculó el rendimiento de recuperación del aceite saponificado en el proceso de hidrólisis.

\subsection{Acidulación para la recuperación de ácidos grasos}

En esta parte del proceso, se llevó a cabo un diseño experimental $3^{2}$ con dos repeticiones en el punto central, en el cual se varió la concentración de ácido sulfúrico con valores de 25, 50 y 75 \% $\mathrm{v} / \mathrm{v}$; adicionalmente, se varió la temperatura del proceso con valores de 20,40 y $60{ }^{\circ} \mathrm{C}$. Para el proceso, la solución de $\mathrm{H}_{2} \mathrm{SO}_{4}$ fue añadida en una cantidad igual a la relación estequiométrica para la neutralización del $\mathrm{NaOH}$ usada en la etapa de la hidrólisis básica (Harting et al., 2010). A continuación, la mezcla se agitó constantemente a 30 oscilaciones por minuto durante 10 min en un baño térmico marca Selecta modelo Unitronic Or, a las temperaturas del diseño experimental (Sánchez, 2006). Como consecuencia de la reacción, se formaron dos fases. Una fase aceitosa, en la cual se encuentran los ácidos grasos liberados, la cual flotó y se ubicó en la parte superior de la solución y una fase acuosa que contuvo sales minerales y agua que se ubicó en la parte inferior de la solución. 


\subsection{Cromatografía de gases}

\subsubsection{Esterificación de los ácidos grasos}

El proceso de esterificación se llevó a cabo como paso previo a la cromatografía de gases, para aumentar la volatilidad de los ácidos grasos, de manera que sus concentraciones puedan ser determinadas. Se esterificaron todas las muestras aciduladas en las diferentes condiciones experimentales.

Para esto, se colocaron 2 gotas de las muestras aciduladas, en un tubo de ensayo con tapa, al cual se añadió $1 \mathrm{~mL}$ de una solución 0,5 M de $\mathrm{KOH}$ disuelta en metanol. El recipiente se tapó y la solución resultante fue calentada en baño térmico, con la ayuda de una plancha de calentamiento marca Corning, hasta que se alcanzó la ebullición, que se mantuvo durante $10 \mathrm{~min}$. Posteriormente, se agregaron 0,4 $\mathrm{mL}$ de una solución 1:4 v/v de $\mathrm{HCl}$ al 37\%, en metanol. Esta mezcla se mantuvo en ebullición durante $25 \mathrm{~min}$ en baño térmico. Luego, la mezcla fue enfriada y se agregaron $2 \mathrm{~mL}$ de agua destilada (Metcalfe y Schmitz, 1961). A continuación, se realizó una primera extracción, donde se colocaron $3 \mathrm{~mL}$ de éter de petróleo en el recipiente con la muestra y se agitó vigorosamente, se dejó en reposo hasta que se formaron dos fases. La fase etérea ubicada en la parte superior fue recuperada con la ayuda de una pipeta Pasteur y se colocó en un vaso de precipitación. De la misma manera, se realizó una segunda extracción a partir de la fase inferior de la solución, para obtener mayor cantidad de muestra de la fase etérea. Posteriormente, se añadió sulfato de sodio anhidro para evitar la presencia de agua residual en la fase etérea recuperada. Finalmente, esta solución fue filtrada con un filtro para cromatografía de 0,45 $\mu \mathrm{m}$ y se colocó en un tubo de ensayo. La solución se dejó en reposo durante $15 \mathrm{~h}$ a temperatura ambiente, donde se concentró hasta que se obtuvieron $2 \mathrm{~mL}$ de la misma (Metcalfe y Schmitz, 1961).

\subsubsection{Condiciones para la cromatografía de gases}

Con la ayuda de una jeringuilla para cromatografía, se tomaron $0,5 \mu \mathrm{L}$ de la solución de la muestra esterificada y se inyectaron en una columna HP-23 (cis/FAME Column) de $30 \mathrm{~m}$ de longitud, 0,32 mm de diámetro y 0,25 $\mu \mathrm{m}$ de espesor, en un cromatógrafo marca PerkinElmer modelo Clarus 500, a una temperatura inicial del horno de $150^{\circ} \mathrm{C}$, con una rampa de $15^{\circ} \mathrm{C} / \mathrm{min}$, hasta $210^{\circ} \mathrm{C}$. La corrida de la muestra en el equipo duró $56 \mathrm{~min}$. La temperatura de inyección de los compuestos fue $250{ }^{\circ} \mathrm{C}$, la temperatura del detector fue $260^{\circ} \mathrm{C}$ y la velocidad de inyección de helio fue de $35 \mathrm{~cm} / \mathrm{s}$ (Piras et al., 2013). Las condiciones descritas fueron aplicadas también a un estándar, con la finalidad de comparar los picos obtenidos en los distintos cromatogramas. Dentro de esta comparación se evaluaron las áreas de los picos, las cuales permitieron determinar las concentraciones de los ácidos grasos en las muestras estudiadas. 


\section{Resultados y Discusión}

\subsection{Caracterización química proximal de las cabezas de sardina (Opisthonema libertate)}

En la Tabla 1 se representan los valores correspondientes a la caracterización de la especie en estudio mediante un análisis químico proximal. La humedad dentro de la muestra constituyó el valor más elevado en la composición proximal correspondiente a un 67,23 $\pm 1,3 \%$ del total analizado. La composición mayoritaria de agua se debe a la naturaleza del producto, razón por la cual fue importante mantener un manejo adecuado en la cadena de frío desde su origen hasta su tratamiento. Asimismo, el contenido de minerales y materia inorgánica, representado por el porcentaje de cenizas, presentó un valor de 8,50 \pm 0,22\%, el cual fue mayor al obtenido para los residuos de otras especies de sardina, como la Sardinella aurita, que presentó 1,64 \% y la Sardina pilchardus con un 1,90 \% (Kechaou et al., 2009; Valls y Paredes, 2010). Esta peculiaridad se da debido a que la especie en estudio involucra únicamente cabezas; mientras que las especies comparadas involucran además vísceras y el cuerpo de los pescados. Además, se podría considerar que el mayor contenido de cenizas en Opisthonema libertate pudo deberse al hábitat del que la especie en estudio procede, correspondiente a agua de mar; mientras que las otras especies mencionadas habitan en ecosistemas de agua dulce (FAO, 1999).

Tabla 1. Resultados de la composición química proximal de cabezas de sardina (Opisthonema libertate)

\begin{tabular}{ccccc}
\hline & $\begin{array}{c}\text { Humedad } \\
(\%)\end{array}$ & $\begin{array}{c}\text { Cenizas } \\
(\%)\end{array}$ & $\begin{array}{c}\text { Extracto } \\
\text { Etéreo } \\
(\%)\end{array}$ & $\begin{array}{c}\text { Proteína } \\
(\%)\end{array}$ \\
\hline Cabezas & $67,23 \pm$ & $8,50 \pm$ & $5,58 \pm$ & $17,55 \pm$ \\
de sardina & 1,38 & 0,22 & 0,13 & 0,24 \\
\hline
\end{tabular}

El porcentaje correspondiente al extracto etéreo fue 5,58 $\pm 0,13 \%$. Al comparar este valor con el de otras especies de pescado del tipo azul como el Epinephelus coioides, cuyo porcentaje de lípidos es 3,73\%, la Sardina pilchardus con $4,89 \%$ y la Sardinella aurita con 3,44 \%, se observó que la sardina presentó una mayor cantidad de aceite susceptible de recuperación, lo que probablemente indicaría mayor presencia de ácidos grasos (Kechaou et al., 2009; Tawfik, 2010; Valls y Paredes, 2010).

Finalmente, el contenido de proteína en la muestra correspondió a un valor de 17,55 \pm 0,24 \% e indica la presencia de nutrientes proteicos. Estos nutrientes se encuentran asociados a los lípidos, por lo que la interacción lípido proteica es la más influyente en la selección del método de extracción de aceite, debido a la necesidad de obtener el mismo en su estado más puro. El valor obtenido guarda similitud con otras especies, como la Sardinella aurita, cuyo porcentaje es de 16,87\% (Valls y Paredes, 2010). 


\subsection{Hidrólisis Básica}

Dentro del proceso de obtención de aceite saponificado, las cabezas de sardina fueron sometidas a un tratamiento de hidrólisis básica mediante el uso de $\mathrm{NaOH}$ en diferentes concentraciones. Dichas concentraciones fueron distintas con el fin de identificar las condiciones idóneas, que maximicen la recuperación de aceite saponificado. Como se observa en la Figura 3, a medida que aumenta la concentración de $\mathrm{NaOH}$ en la solución hidrolizante, aumenta el rendimiento de recuperación del aceite saponificado.

El análisis estadístico reportado por el software Statgraphics Centurion XVI, con análisis de varianza ANOVA manifestó diferencias significativas $(p<0,05)$. Al utilizar concentraciones de 0,$05 ; 0,10$ y 0,50 $\mathrm{M}$, el rendimiento de recuperación de aceite saponificado fue estadísticamente similar en todos los casos, con una ligera tendencia creciente conforme el incremento de las concentraciones. Sin embargo, al utilizar una concentración de 1,00 M, la diferencia fue significativa con respecto a las otras concentraciones utilizadas, con un porcentaje de recuperación del 3,64 \%. Por este motivo, la solución 1,00 M fue utilizada para continuar con el desarrollo del proyecto. Este resultado estuvo en concordancia con lo descrito por Jayasighe et al. (2013), cuyo rendimiento estuvo entre el 2 \% y el $3 \%$. Por lo tanto, el procedimiento empleado para la recuperación de aceite presentó un rendimiento similar al obtenido en procesos que involucran únicamente variaciones térmicas, como es el caso de Jayasighe et al. (2013). Sin embargo, la metodología propuesta permitió saponificar simultáneamente el aceite extraído, lo que representa una ventaja respecto a otras metodologías, en las cuales la saponificación es llevada a cabo posteriormente.

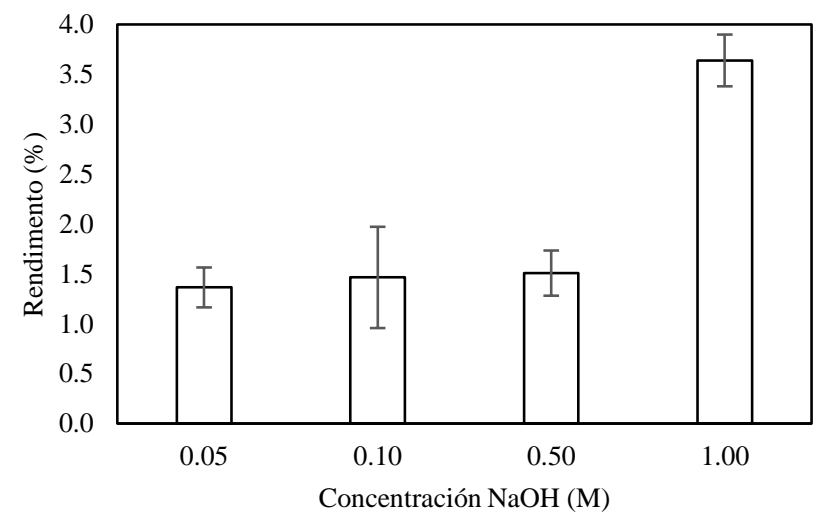

Figura 3. Porcentaje de aceite saponificado recuperado

La razón por la que el porcentaje de rendimiento fue mayor para la concentración $1 \mathrm{M}$ de $\mathrm{NaOH}$, se debe al uso de una base fuerte, que a mayores concentraciones aumentó la carga iónica de la solución y el pH de la misma, lo que pudo influir en el proceso de desnaturalización de la proteína al volverla más soluble en agua. En consecuencia, se favoreció la obtención del aceite saponificado (Giraldo et al., 2010; Macarulla y Goñi, 2002; Velásquez, 2006). 
Respecto a la coloración de la muestra, se pudo notar que a mayores concentraciones de $\mathrm{NaOH}$, el aceite saponificado tomó una tonalidad amarilla más intensa, propia de sustancias grasas, como se observa en la Figura 4 (Caridad et al., 2016); mientras que, a menores concentraciones, las muestras tomaron un color café-rojizo, característico de impurezas, como proteína animal. Cabe mencionar que en la figura se observa la división de dos fases, lo cual no corresponde a diferentes sustancias, sino al estado líquido y sólido de la muestra, lo cual se dio como resultado de la temperatura de almacenamiento. De manera que la fase inferior correspondió a una primera extracción, donde el aceite saponificado fue almacenado en congelación luego de su extracción; mientras que la fase superior representa el aceite saponificado recién extraído.

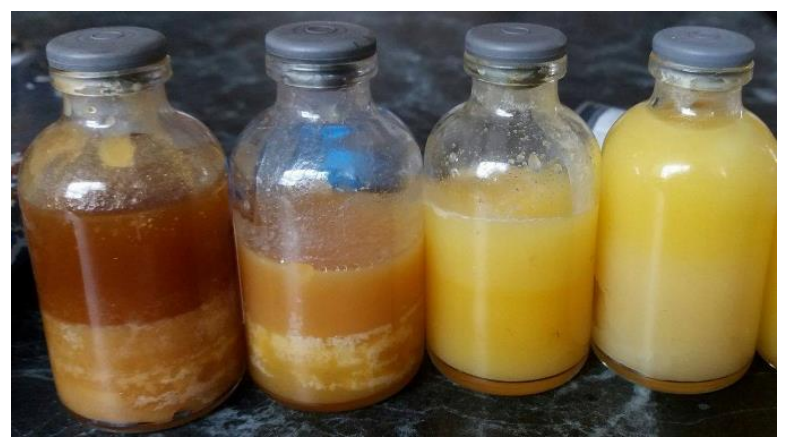

Figura 4. Aceite recuperado a diferentes concentraciones de $\mathrm{NaOH}$, de izquierda a derecha se empleó 0,05 M, 0,10 M, 0,50 M y 1,00 M

Se analizó el contenido de proteína en la muestra obtenida a $1 \mathrm{M}$ de $\mathrm{NaOH}$, para descartar que fracciones remanentes de proteína se encontraran en la muestra. El valor del contenido de proteína correspondió a 0,19 $\pm 0,04 \%$, el cual evidenció una remoción casi total de proteína en comparación con la cantidad de proteína obtenida inicialmente en las cabezas de sardina. De esta forma, se pudo concluir que el tratamiento con $\mathrm{NaOH}$, a una concentración $1 \mathrm{M}$, permitió la mayor extracción del aceite saponificado, en términos de cantidad y pureza.

\subsection{Acidulación}

Se determinó la influencia de los factores concentración de ácido sulfúrico y temperatura, al observar la concentración de los ácidos grasos en cada una de las condiciones experimentales, medida en $(\mu \mathrm{g} / \mathrm{mL})$. Para el análisis de los datos, se dividieron los ácidos grasos en ácidos grasos saturados, monoinsaturados y poliinsaturados. Los ácidos grasos poliinsaturados comprenden a los omega-3 y omega-6, por lo tanto, este grupo de ácidos grasos fue el de mayor importancia para determinar las mejores condiciones del proceso. En la Figura 5, se presenta un diagrama de Pareto que evalúa la influencia de las condiciones experimentales utilizadas, con el fin de identificar qué factores inciden significativamente en la investigación. Se puede observar que la concentración de ácido sulfúrico fue el único factor significativo dentro de la experimentación. Además, se indica que la temperatura no presentó una influencia estadísticamente significativa. Del mismo modo, al analizar ambas condiciones en conjunto, se distingue que la interacción entre los dos factores estudiados tampoco fue significativa. El efecto positivo de la concentración de ácido sulfúrico dentro 
del proceso implicó que a medida que aumentara la concentración del mismo, la recuperación de ácidos grasos poliinsaturados fuera mayor. Esto se explica en parte, debido a la interacción del ácido como catalizador dentro de la reacción de sustitución, la cual puede observarse en la Figura 2. De esta forma, a medida que la concentración del ácido aumentó, la extracción de los ácidos grasos por procesos de sustitución se pudo ver favorecida (Yúfera, 2007).

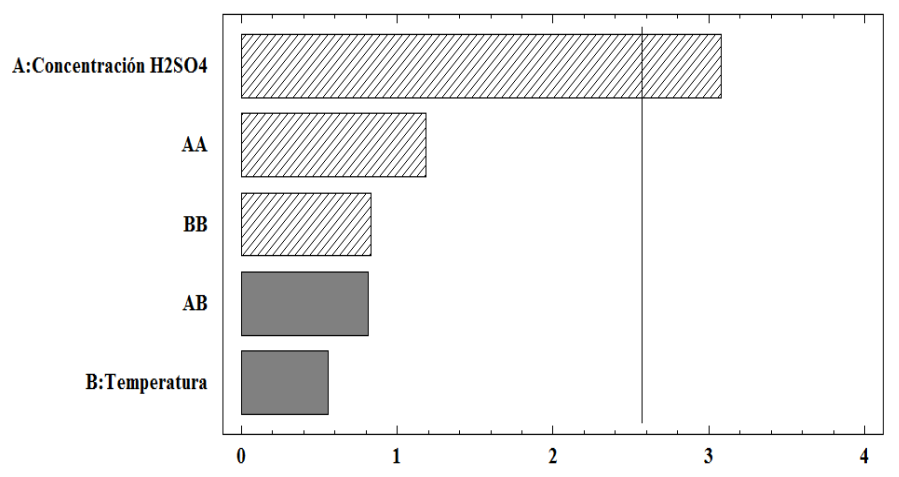

Figura 5. Diagrama de Pareto para la concentración de ácidos grasos poliinsaturados presentes en la muestra respecto a las condiciones de trabajo utilizadas

Para establecer cuál fue la condición óptima dentro del proceso, se analizó la Figura 6, donde se puede apreciar que la mayor superficie se encuentra orientada hacia la concentración de $75 \% \mathrm{v} / \mathrm{v}$ de ácido sulfúrico y $20^{\circ} \mathrm{C}$, con una recuperación total de ácidos grasos poliinsaturados de 83,26 $\mu \mathrm{g} / \mathrm{mL}$, que correspondió a la mayor cantidad recuperada en relación con las demás condiciones analizadas.

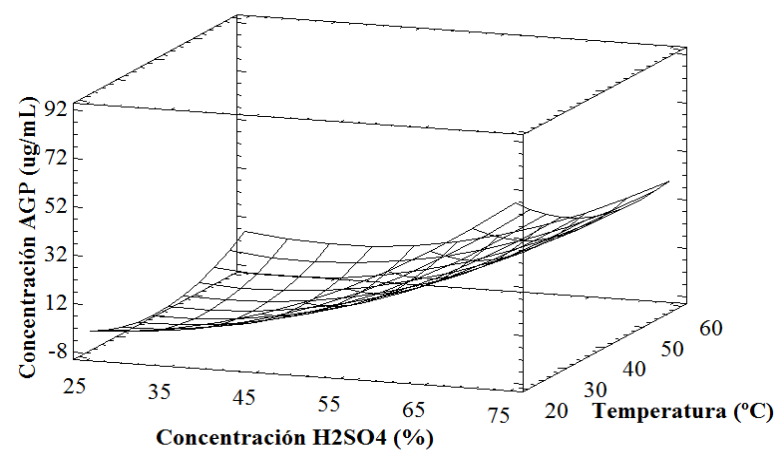

Figura 6. Superficie de respuesta estimada para la concentración de ácidos grasos poliinsaturados presentes en la muestra respecto a las condiciones de trabajo utilizadas

A pesar que la temperatura no mostró ser un factor significativo en el proceso, la mejor condición se presentó a $20^{\circ} \mathrm{C}$, la cual correspondió a la menor temperatura dentro del rango estudiado. Esto pudo deberse a que mayores temperaturas implican una aceleración en las reacciones de deterioro de la estructura molecular de los ácidos grasos, entre las que se pueden destacar procesos de hidrólisis y oxidación (Marcano et al., 2007; Zamorano et al., 2013). La interacción entre la fuerza del ácido en conjunto con las altas temperaturas del proceso pudieron ser la causa de reacciones de degradación del aceite. En la Tabla 3, se observa una recopilación de los ácidos grasos obtenidos bajo la mejor condición experimental $\left(20{ }^{\circ} \mathrm{C}\right.$ y $75 \% \mathrm{v} / \mathrm{v}$ de $\left.\mathrm{H}_{2} \mathrm{SO}_{4}\right)$. Los ácidos grasos 
saturados obtuvieron el mayor porcentaje de recuperación, con un 82,55 \% del total recuperado. Dentro de dicha categoría, es importante destacar la presencia ácido palmítico C 16:0 con una concentración correspondiente a $970,10 \mu \mathrm{g} / \mathrm{mL}$, consistente con lo reportado en investigaciones similares (González et al., 2013; Valls y Paredes, 2010).

Asimismo, el contenido de ácidos grasos monoinsaturados fue del $13,72 \%$ del total recuperado. El ácido mayoritario en este grupo fue el ácido oleico C 18:1n9c, con una concentración de 152,35 $\mu \mathrm{g} / \mathrm{mL}$, la cual, al ser comparada con otros resultados obtenidos en estudios similares, cuya concentración fue $395,36 \mu \mathrm{g} / \mathrm{mL}$, permitió observar que se trató del ácido graso monoinsaturado de mayor proporción en este tipo de muestras (González et al., 2013; Valls y Paredes, 2010).

Tabla 3. Perfil de ácidos grasos en la muestra acidulada a $20{ }^{\circ} \mathrm{C}$ y $75 \% \mathrm{v} / \mathrm{v}$ de $\mathrm{H}_{2} \mathrm{SO}_{4}$

\begin{tabular}{|c|c|}
\hline \multirow[b]{2}{*}{ Ácido graso } & \multirow[b]{2}{*}{$\begin{array}{c}\text { Concentración } \\
\text { ácidos grasos } \\
(\mu \mathrm{g} / \mathrm{mL})\end{array}$} \\
\hline & \\
\hline Ác. Butanoico, C 4:0 & 182,63 \\
\hline Ác. Caproico, C 6:0 & 47,84 \\
\hline Ác. Caprílico, C 8:0 & 5,75 \\
\hline Ác. Cáprico, C 10:0 & 54,29 \\
\hline Ác. Undecanoico, C 11:0 & 5,46 \\
\hline Ác. Láurico, C 12:0 & 25,06 \\
\hline Ác. Tridecanoico, C 13:0 & 166,66 \\
\hline Ác. Mirístico, C 14:0 & 19,24 \\
\hline Ác. Palmítico, C 16:0 & 970,10 \\
\hline Ác. Margárico, C 17:0 & 64,45 \\
\hline Ác. Esteárico, C 18:0 & 247,78 \\
\hline Ác. Tricosanoico, C 23:0 & 50,99 \\
\hline Ác. Lignocérico, C 24:0 & 3,66 \\
\hline TOTAL AGS & 1843,94 \\
\hline$\%$ TOTAL AGS & 82,55 \\
\hline Ác. Misitoleico, C 14:1n5 & 63,05 \\
\hline Ác. Pentadecanoico cis-10, C 15:1n5 & 44,22 \\
\hline Ác. Palmitoleico, C 16:1n7 & 46,53 \\
\hline Ác. Oleico, C 18:1n9c & 152,35 \\
\hline Ác. Eicosanoico cis-11, C 20:1n9 & 0,35 \\
\hline TOTAL AGM & 306,50 \\
\hline$\%$ TOTAL AGM & 13,72 \\
\hline Ác. Linoleico, C 18:2n6c & 9,91 \\
\hline Ác. Gama-linoleico, C 18:3n6 & 0,23 \\
\hline $\begin{array}{l}\text { Ác. Eicosapentaenoico EPA, C } \\
20: 5 n 3\end{array}$ & 73,12 \\
\hline TOTAL AGP & 83,26 \\
\hline$\%$ TOTAL AGP & 3,73 \\
\hline
\end{tabular}

Finalmente, los ácidos grasos poliinsaturados, objeto de este estudio, presentaron un porcentaje equivalente al 3,73 \% del total. Se recuperaron: ácido linoleico C 18:2n6c (omega-6), ácido gama 
linoleico C 18:3n6 (omega-6) y ácido eicosapentaenoico EPA C 20:5n3 (omega-3). Dentro de estos, el de mayor cantidad fue el EPA con $36,63 \mu \mathrm{g} / \mathrm{mL}$.

Se observó que Opisthonema libertate, especie empleada en este estudio, no presentó porcentajes de contenido de ácidos grasos poliinsaturados similares con los reportados en otros estudios, donde alcanzan valores entre 31,58 \% a 37,00 \% (Valls y Paredes, 2010). Esto puede deberse a las características genéticas de la especie y además, a pesar que la sardina pertenece al grupo de peces azules, que se caracterizan por el alto contenido de ácidos grasos omega-3 y omega-6, un factor importante a considerar es la temperatura del medio donde habitan. De forma que, a menor temperatura ambiente, mayor es la cantidad de ácidos grasos encontrados en los peces (González et al., 2013). De igual manera, influye la estación del año en la que los peces son capturados, de tal manera que en verano hay una menor cantidad de ácidos grasos susceptibles de recuperación, es decir, cuando la temperatura del ambiente donde habitan es más cálida, como en el caso de las muestras analizadas en este trabajo (Pariona, 2008; Salas et al., 2002).

La Figura 7 presenta el cromatograma obtenido en la mejor condición experimental, en el cual se pueden observar los picos característicos para cada ácido graso presente en la muestra. La identificación de la mayoría de los ácidos grasos por cromatografía se dio en tiempos relativamente cortos de 1,5 a 6 min. La mayoría de los ácidos grasos recuperados a menores tiempos correspondieron a aquellos que poseen su cadena completamente saturada. Igualmente, el ácido palmítico posee el pico más alto dentro del cromatograma en estudio, con un tiempo de extracción de aproximadamente 3,6 min.

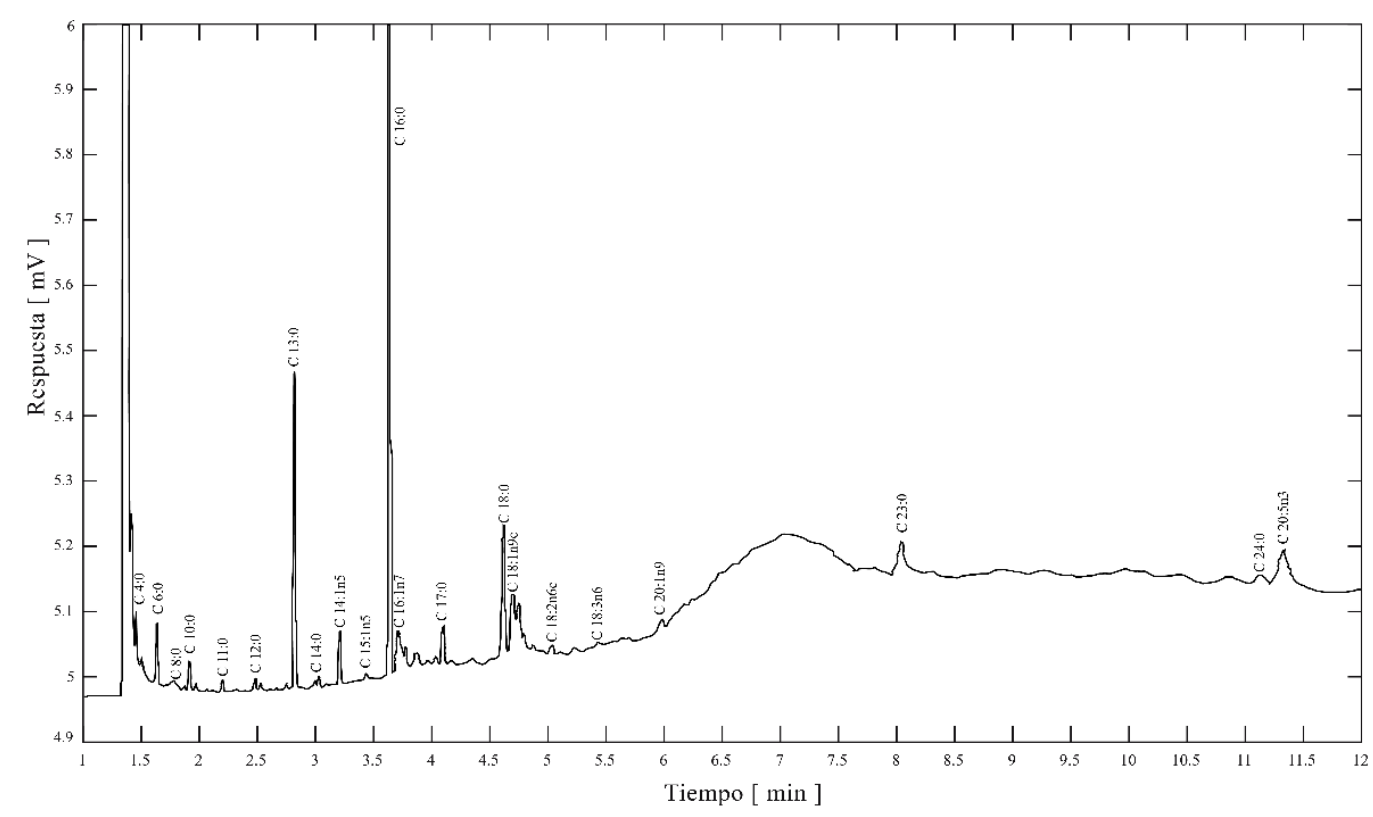

Figura 7. Cromatograma de aceite acidulado a $75 \%$ de $\mathrm{H}_{2} \mathrm{SO}_{4}$ y $20{ }^{\circ} \mathrm{C}$ 
A medida que los tiempos aumentan, se puede observar que los ácidos grasos de cadena más larga son identificados, como por ejemplo los ácidos grasos poliinsaturados que se identifican en tiempos superiores a los $11 \mathrm{~min}$. Finalmente, al realizar una comparación del cromatograma obtenido con otros presentados en estudios similares, se observa que el comportamiento de los picos es similar (Salas et al., 2002).

\section{Conclusiones y Recomendaciones}

El análisis proximal de las cabezas de sardina (Opisthonema libertate) permitió determinar un contenido de humedad del $67,23 \pm 1,38 \%, 8,50 \pm 0,22 \%$ en cenizas, $5,58 \pm 0,13 \%$ de extracto etéreo y $17,55 \pm 0,24 \%$ de proteína.

En el proceso de hidrólisis básica, al utilizar concentraciones de 0,05; 0,10 y 0,50 $\mathrm{M}$ de $\mathrm{NaOH}$, el rendimiento de recuperación de aceite saponificado fue estadísticamente similar en todos los casos con una ligera tendencia creciente al aumentar la concentración. La concentración de $\mathrm{NaOH}$ con la cual se obtuvo la mayor recuperación de aceite saponificado correspondió a un valor de $1 \mathrm{M}$, con un rendimiento correspondiente a un 3,64\%.

En el proceso de acidulación, la mejor condición experimental se dio en un valor de $75 \%$ de concentración de ácido sulfúrico y una temperatura equivalente a $20{ }^{\circ} \mathrm{C}$. A dichas condiciones, la concentración de ácidos grasos poliinsaturados correspondió a 83,26 $\mu \mathrm{g} / \mathrm{mL}$.

El efecto de la interacción entre la temperatura y la concentración de ácido sulfúrico dentro del proceso fue no significativo. Mientras que, la concentración de ácido sulfúrico si influyó significativamente en la recuperación de ácidos grasos.

De acuerdo con su saturación, los porcentajes de ácidos grasos obtenidos en la mejor condición experimental fueron $82,5 \%$ para los saturados, $13,7 \%$ para los monoinsaturados y $3,8 \%$ para los poliinsaturados del total recuperado.

Dentro de los ácidos grasos saturados, el de mayor recuperación fue el ácido palmítico con una concentración correspondiente a $970,10 \mu \mathrm{g} / \mathrm{mL}$. Mientras que, para los ácidos grasos monoinsaturados, el de mayor recuperación fue el ácido oleico con una concentración de 152,35 $\mu \mathrm{g} / \mathrm{mL}$.

Los ácidos grasos poliinsaturados obtenidos fueron: ácido linoléico (omega-6), ácido gama linoleico (omega-6) y ácido eicosapentaenoico EPA (omega-3). Dentro de estos, el de mayor concentración fue el EPA con $73,12 \mu \mathrm{g} / \mathrm{mL}$. 


\section{Bibliografía}

Association of Official Analytical Methods. Official Methods 920.153: Ash of Meat: Official Methods of Analysis of AOAC International, 2007.

Association of Official Analytical Methods. Official Methods 981.10: Crude Protein in Meat: Official Methods of Analysis of AOAC International, 2007.

Association of Official Analytical Methods. Official Methods 960.39: Fat-Crude or Ether Extract in Meat: Official Methods of Analysis of AOAC International, 2007.

Association of Official Analytical Methods. Official Methods 950.46: Moisture in Meat: Official Methods of Analysis of AOAC International, 2007.

Association of Official Analytical Methods. Official Methods 920.160: Saponification Number: Official Methods of Analysis of AOAC International, 2007.

Atinafu, D., \& Bedemo, B. (2011). Estimation of total free fatty acid and cholesterol content in some commercial edible oils in Ethiopia, Bahir DAR. Journal of Cereals and Oilseeds, 2(5), 71-76. doi: $10.5897 / J C O 11.025$

Baião, N. C., \& Lara, L. J. C. (2005). Oil and fat in broiler nutrition. Revista Brasileira de Ciência Avícola, 7(3), 129-141.

Caprioli, G., Giusti, F., Ballini, R., Sagratini, G., Vila-Donat, P., Vittori, S., \& Fiorini, D. (2016). Lipid nutritional value of legumes: Evaluation of different extraction methods and determination of fatty acid composition. Food chemistry, 192, 965-971. doi:10.1016/j.foodchem.2015.07.102

Caridad, M., Fernández, M., Castiñeira, M., Rodríguez, J., Romero, J., \& Márquez, T. (2016). Evaluación y establecimiento de las especificaciones de calidad del pool de aceite de hígado de tiburón. Ars Pharmaceutica, 55(1), 23-29.

Cermak, S. C., Kenar, J. A., \& Evangelista, R. L. (2012). Distillation of natural fatty acids and their chemical derivatives. INTECH Open Access Publisher.

FAO. (1999). El pescado fresco: su calidad y cambios de calidad. Recuperado de: http://www.fao.org/docrep/v7180s/v7180s05.htm (febrero, 2017).

FAO. (2014). El estado mundial de la pesca y la acuicultura. Recuperado de: http://www.fao.org/3/ai3720s.pdf (febrero, 2016).

Gatica, A. (2011). Ácidos grasos EPA y DHA y su vital importancia en la Nutrición Humana. Indualimentos. Instituto de Nutrición y Tecnología de Alimentos (INTA). Universidad de Chile. Santiago de Chile, 58-60. 
Gargaud, M. (2011). Encyclopedia of astrobiology (Vol. 3). Springer Science \& Business Media. Bordeaux, Francia.

Gellman, M., \& Turner, J. R. (2013). Encyclopedia of Behavioral Medicine. Springer. Dong Nai, Vietnam.

Giraldo, G. A. G., Chamorro, N. L., \& Doria, C. M. M. (2010). Laboratorio de Bioquímica: Una Visión Práctica. ELIZCOM SAS. Armenia, Colombia.

González, M. I. C., Rodríguez, A. G. M., \& Gómez, C. G. (2013). Perfil de ácidos grasos de diversas especies de pescados consumidos en México. Revista de Biología Tropical, 61(4), 1981-1998.

Gracia, M. D. (2010). Esterificación de colofonia con glicerina: estudio cinético y fenomenológico. Universidad Complutense de Madrid, Servicio de Publicaciones.

Harting, T., Díaz M., \& Markovits, A. (2010). Proceso para la obtención de un concentrado de esteres de ácidos eicopentaenoico y docosahexaenoico. WO 2010/139085. Múnich: Oficina de Patentes Europeas.

Hordur, K., \& Necla, D. (2003). Functional Fish Protein Ingredients from Fish Species of Warm and Temperate Waters: Comparison of Acid- and Alkali-Aided Processing vs. Conventional Surimi Processing. Advances in Seafood Byproducts 2002 Conference Proceedings, Anchorage, Estados Unidos. Recuperado de: http://nsgd.gso.uri.edu/aku/akuw02001/akuw02001_full.pdf (enero, 2016).

IFFO. (2008). Los Omega-3 más saludables EPA y DHA se encuentran principalmente en el aceite y la harina de pescado. Recuperado de: http://www.iffo.net/es/system/files/DPSP4.pdf (enero, 2016).

Iso, H., Kobayashi, M., Ishihara, J., Sasaki, S., Okada, K., Kita, Y., \& Tsugane, S. (2006). Intake of fish and $\mathrm{n} 3$ fatty acids and risk of coronary heart disease among Japanese. Circulation, 113(2), 195-202.

Jayasinghe, P., Adeoti, I., \& Hawboldt, K. (2013). A study of process optimization of extraction of oil from fish waste for use as a low-grade fuel. Journal of the American Oil Chemists' Society, 90(12), 1903-1915. doi: 10.1007/s11746-013-2321-1

Karasek, F. W., \& Clement, R. E. (2012). Basic gas chromatography-mass spectrometry: principles and techniques. Elsevier. Ontario, Canada.

Kechaou, E. S., Dumay, J., Donnay-Moreno, C., Jaouen, P., Gouygou, J. P., Bergé, J. P., \& Amar, R. B. (2009). Enzymatic hydrolysis of cuttlefish (Sepia officinalis) and sardine (Sardina 
pilchardus) viscera using commercial proteases: effects on lipid distribution and amino acid composition. Journal of bioscience and bioengineering, 107(2), 158-164.

Larsen, R., Eilertsen, K., \& Elvevoll, E. (2011). Health benefits of marine foods and ingredients. Biotechnology Advances, 29(5), 508-518. doi:10.1016/j.biotechadv.2011.05.017

Macarulla, J. M., \& Goñi, F. M. (2002). Biomoléculas: lecciones de bioquímica estructural. Reverté. Bilbao, España.

MAE. (2013). Estudio de potenciales impactos y vulnerabilidad relacionada con las sustancias químicas y tratamiento de desechos peligrosos en el sector productivo del Ecuador. Recuperado de: http://www.ambiente.gob.ec/wpcontent/uploads/downloads/2013/03/PART11.pdf (Febrero, 2016).

Maqsood, S., \& Benjakul, S. (2010). Comparative studies of four different phenolic compounds on in vitro antioxidative activity and the preventive effect on lipid oxidation of fish oil emulsion and fish mince. Food Chemistry, 119(1), 123-132. doi:10.1016/j.foodchem.2009.06.004

Marcano, E. S., Douglas, B., Marín, G., \& Moreno, H. (2007). Cinética de deterioro del aceite de la semilla de la palma Coroba (Attalea maripa). SABER. Revista Multidisciplinaria del Consejo de Investigación de la Universidad de Oriente, 19(2), 172-182.

Mercantili, L., Davis, F., \& Higson, S. (2012). Ultrasonic Initiation of the Alkaline Hydrolysis of Triglycerides (Saponification) Without Phase Catalysis. AOCS, 17, 133-141. doi: $10.1007 / \mathrm{s} 11743-013-1450-8$

Metcalfe, L., \& Schmitz, A. (1961). The rapid preparation of fatty acid esters for gas chromatographic analysis. Analytical Chemistry, 33(3), 363-364. doi: 10.1021/ac60171a016

McMurry, J., Química orgánica. Cengage Learning Editores, 2012. México DF., México

Mora, R. J. (2002). Soporte nutricional especial. Ed. Médica Panamericana. Bogotá, Colombia

Nielsen, S. (2003). Food Analysis. West Lafayette, Estados Unidos: Kluwer Academic/ Plenum Publishers.

Nolsøe, H., \& Undeland, I. (2009). The acid and alkaline solubilization process for the isolation of muscle proteins: state of the art. Food and Bioprocess Technology, 2(1), 1-27. doi: $10.1007 / \mathrm{s} 11947-008-0088-4$

Pariona, N. (2008). Obtención de los ácidos grasos del aceite de la Plukenetia volubilis L." Sacha Inchi" para la utilización en la industria y estudio fitoquímico cualitativo de la almendra. 
Piras, A., Rosa, A., Marongiu, B., Porcedda, S., Falconieri, D., Dessì, M. A., \& Koca, U. (2013). Chemical composition and in vitro bioactivity of the volatile and fixed oils of Nigella sativa L. extracted by supercritical carbon dioxide. Industrial Crops and Products, 46, 317-323.

ProEcuador. (2015). Análisis sectorial: Sardinas. Recuperado de: http://www.proecuador.gob.ec/wpcontent/uploads/2015/08/PROEC_AS2015_SARDINAS.pdf (Enero, 2016).

Rubio-Rodríguez, N., Sara, M., Beltrán, S., Jaime, I., Sanz, M. T., \& Rovira, J. (2012). Supercritical fluid extraction of fish oil from fish by-products: A comparison with other extraction methods. Journal of Food Engineering, 109(2), 238-248. doi:10.1016/j.jfoodeng.2011.10.011

Sahena, F., Zaidul, I. S. M., Jinap, S., Yazid, A. M., Khatib, A., \& Norulaini, N. A. N. (2010). Fatty acid compositions of fish oil extracted from different parts of Indian mackerel (Rastrelliger kanagurta) using various techniques of supercritical CO 2 extraction. Food Chemistry, 120(3), 879-885. doi:10.1016/j.foodchem.2009.10.055

Salas, A., Ayala, M. E., \& Albrecht, M. (2002). Contenido de EPA y DHA en aceite crudo de pescado producido en el Perú durante el período 1996-2000. Cienc. Tecnol. Aliment, 3(5), 283-287.

Sánchez, J. (2006). Extracción de Ácidos Grasos insaturados y obtención del Omega 3 de los residuos industriales pesqueros utilizando tecnologías más limpias. (Proyecto de titulación previo a la obtención del título de Biólogo). Recuperado de la base de datos Academia

Sanz, F. (2009). La nutrición y alimentación en piscicultura. Madrid, España: Publicaciones científicas y tecnológicas de la Fundación Observatorio Español de Acuicultura.

Scrimgeour, C. (2005). Chemistry of fatty acids. Scottish crop research institute. Dundee, Scotland. Bailey's Industrial Oil and Fat Products, Sixth Edition, Six Volume Set. Edited by Fereidoon Shahidi, 2. Dundee, Escocia

Tawfik, M. (2010). Proximate composition and fatty acids profiles in most common available fish species in Saudi market. Asian Journal of Clinical Nutrition, 2(3), 157-164.

Tobón, F. A., Román, M. O., Molina, S., \& Bothert, J. P. (2016). Determinación del perfil de ácidos grasos de la secreción de la glándula uropigial de la Coturnix coturnix japonica (codorniz doméstica). Revista Colombiana de Ciencias Pecuarias, 15(2), 169-179.

Valenzuela, A., Sanhueza, J., \& De la Barra, F. (2012). El aceite de pescado: ayer un desecho industrial, hoy un producto de alto valor nutricional. Revista Chilena de Nutrición, 39(2), 201209. http://dx.doi.org/10.4067/S0717-75182012000200009 
Valenzuela, R., Tapia, G., González, M., \& Valenzuela, A. (2011). Ácidos grasos omega-3 (EPA y DHA) y su aplicación en diversas situaciones clínicas. Revista Chilena de Nutrición, 38(3), 356-367. http://dx.doi.org/10.4067/S0717-75182011000300011

Valls, J. E., \& Paredes, A. (2010). Caracterización física y química de la sardina (Sardinella aurita). Revista Científica, 20(5), 546-554.

Valverde, L. M., Moreno, P. A. G., Callejón, M. J. J., Cerdán, L. E., \& Medina, A. R. (2013). Concentration of eicosapentaenoic acid (EPA) by selective alcoholysis catalyzed by lipases. European Journal of Lipid Science and Technology, 115(9), 990-1004. doi:10.1002/ejlt.201300005

Velásquez, G. (2006). Fundamentos de alimentación saludable. Universidad de Antioquia. Medellín, Colombia.

Wijayarathne, U., \& Wasalathilake, K. (2014). Aspen Plus Simulation of Saponification of Ethyl Acetate in the Presence of Sodium Hydroxide in a Plug Flow Reactor. Journal of Chemical Engineering \& Process Technology,2014. doi:10.4172/2157-7048.1000205

Yúfera, E. P. (2007). Química orgánica básica y aplicada: de la molécula a la industria (Vol. 2). Reverté. Valencia, España.

Zamorano, M., Martínez, S., \& Medel, J. (2013). Comportamiento del perfil de ácidos grasos de aceites y materias grasas hidrogenadas sometidos a calentamiento prolongado. Revista de la Facultad de Ciencias Agrarias. Universidad Nacional de Cuyo, 45(1), 0-0. 